\title{
Stationary reflection and the universal Baire property
}

\author{
by
}

\author{
Stuart Zoble (Toronto)
}

\begin{abstract}
We show that $\omega_{1}$-Universally Baire self-justifying systems are fully Universally Baire under the Weak Stationary Reflection Principle for Pairs. This involves analyzing the notion of a weakly captured set of reals, a weakening of the Universal Baire Property.
\end{abstract}

Introduction. Woodin has shown that the Weak Stationary Reflection Principle for Pairs $\mathrm{WRP}_{(2)}\left(\omega_{2}\right)$ implies that if every subset of $\omega_{1}$ has a sharp then every subset of $\omega_{2}$ has a sharp. He has shown more generally that under $\operatorname{WRP}_{(2)}\left(\omega_{2}\right)$, if PD holds after collapsing $\omega_{1}$ then it continues to hold after collapsing $\omega_{2}$ (see 9.78 and 9.84 of [11]). Similar arguments appear throughout [7] where stationary reflection is used to lift closure under various operations from $H\left(\omega_{2}\right)$ to $H\left(\omega_{3}\right)$. In this note we attempt to unify these arguments in terms of a weakening of the Universal Baire Property.

We say that a set of reals $A$ is weakly captured at $\kappa$ if there is a $\operatorname{Col}(\omega, \kappa)$ term $\dot{A}$ such that for sufficiently large $\theta$, for a club of countable $H \prec H(\theta)$, and for a comeager set of $g: \omega \rightarrow$ ot $(H \cap \kappa)$,

$$
\pi_{H}(\dot{A})_{g}=A \cap H[g],
$$

where ot $(H \cap \kappa)$ is the order type of $H \cap \kappa$ and $\pi_{H}$ is the transitivization map. This property is a consequence of $A$ having the $\kappa$-Universal Baire Property and it is known not to be equivalent in general (see 3.2 below). It is true however that if the condition "comeager many $g$ " is replaced by "all generic $g$ " then an equivalent property is obtained. Our main result is that under $\mathrm{WRP}_{(2)}(\kappa)$, if a set of reals $A$ is weakly captured at $\omega_{1}$ then it is weakly captured at $\kappa$.

We show further that if $A$ and $\omega^{\omega} \backslash A$ have semiscales whose prewellorderings are weakly captured at $\omega_{1}$ then $A$ is in fact $\kappa$-Universally Baire. Thus, under MM for example, or if $\omega_{2}$ is generically supercompact (see [5]), self-

2000 Mathematics Subject Classification: Primary 03E15; Secondary 03E05.

Key words and phrases: stationary set, stationary reflection, Universal Baire Property. 
justifying systems $\left({ }^{1}\right)$ which are $\omega_{1}$-Universally Baire are fully Universally Baire. Recently the author has shown with Todorcevic that $\omega_{1}$-Universally Baire sets are Universally Baire under the Game Reflection Principle GRP ${ }^{+}$ (see 2.14 below). Todorcevic has shown however that under MM there is a set of reals which is $\omega_{1}$-Universally Baire but not fully Universally Baire, so that some additional hypothesis (such as capturing of scales) is needed for the main theorem here. These and related results will appear in [9].

1. Definitions and preliminary remarks. We think of a real as an element of the Baire space $\omega^{\omega}$ of infinite sequences of integers, and for any set $X$ we endow $X^{\omega}$ with the product topology, giving the set $X$ the discrete topology. For an uncountable set $X$ we denote by $[X]^{\omega}$ the set of countable subsets of $X$, and by $[X]^{\omega_{1}}$ the set of subsets of $X$ of size $\omega_{1}$. A set $C \subseteq[X]^{\omega}$ is a club if it is cofinal (in the $\subseteq$ ordering) and closed under countable increasing unions. A set $S \subseteq[X]^{\omega}$ is stationary if it has nonempty intersection with every club. We will use the following facts about clubs and stationary sets (see $[1,2])$.

Lemma 1.1. Let $X \subseteq Y$ be uncountable, $\theta$ a regular cardinal, and $a \in H(\theta)$.

(1) $\left\{H \in[H(\theta)]^{\omega} \mid a \in H \prec H(\theta)\right\}$ is club in $[H(\theta)]^{\omega}$.

(2) If $S \subseteq[X]^{\omega}$ is stationary and $\psi: S \rightarrow X$ satisfies $\psi(\sigma) \in \sigma$ for every $\sigma \in S$ then $\psi$ is constant on a stationary subset of $[X]^{\omega}$.

(3) If $S \subseteq[Y]^{\omega}$ is stationary then $\{\sigma \cap X \mid \sigma \in S\}$ is stationary in $[X]^{\omega}$.

(4) If $S \subseteq[X]^{\omega}$ is stationary then $\left\{\sigma \in[Y]^{\omega} \mid \sigma \cap X \in S\right\}$ is stationary in $[Y]^{\omega}$.

(5) If $C \subseteq[X]^{\omega}$ is club then there is a function $f: X^{<\omega} \rightarrow X$ such that $C_{f}=\left\{\sigma \in[X]^{\omega} \mid f\left[\sigma^{<\omega}\right] \subseteq \sigma\right\} \subseteq C$.

For an uncountable cardinal $\kappa$, the Weak Reflection Property for Pairs at $\kappa$, denoted $\mathrm{WRP}_{(2)}(\kappa)$, is defined as follows.

DEFINITION 1.2. $\mathrm{WRP}_{(2)}(\kappa)$ is the assertion that for any stationary $S, T \subseteq[\kappa]^{\omega}$ the set of $X \in[\kappa]^{\omega_{1}}$ for which both $S \cap[X]^{\omega}$ and $T \cap[X]^{\omega}$ are stationary in $[X]^{\omega}$ is cofinal in $[\kappa]^{\omega_{1}}$.

In case $\kappa=\omega_{2}$, we may assume that $X \in \omega_{2}$. Work of Baumgartner shows that if $\kappa$ is weakly compact and $G \subset \operatorname{Col}\left(\omega_{1},<\kappa\right)$ is $V$-generic then $V[G] \models \mathrm{WRP}_{(2)}\left(\omega_{2}\right)$ (see [5] for a proof using game reflection). $\mathrm{WRP}_{(2)}(\kappa)$ for higher $\kappa$ is a consequence of MM or the generic supercompactness of $\omega_{2}$ $($ see $[3,5])$. Our reference for the Universal Baire Property is [2].

$\left({ }^{1}\right)$ Closed under complements and prewellorderings of scales. 
Definition 1.3. A set $A \subset \omega^{\omega}$ is $\kappa$-Universally Baire if $f^{-1}(A)$ has the Baire Property in $\kappa^{\omega}$ for any continuous function $f: \kappa^{\omega} \rightarrow \omega^{\omega}$.

We extend this definition to subsets of $\left(\omega^{\omega}\right)^{n}$ for any finite power $n$ using any recursive homeomorphism $f:\left(\omega^{\omega}\right)^{n} \rightarrow \omega^{\omega}$, and let $\Gamma_{\kappa}^{\mathrm{UB}}$ denote the pointclass of $\kappa$-Universally Baire sets. A more useful formulation is given in terms of absolutely complementing trees.

Theorem 1.4 (Feng, Magidor, Woodin [2]). $A \subset \omega^{\omega}$ belongs to $\Gamma_{\kappa}^{\mathrm{UB}}$ if and only if there are trees $S, T \subset(\omega \times \lambda)^{<\omega}$ for some $\lambda$ which project to $A, \omega^{\omega} \backslash A$ respectively and which continue to project to complements after collapsing $\kappa$, that is,

$$
V[G] \models p[S]=\omega^{\omega} \backslash p[T]
$$

whenever $G \subset \operatorname{Col}(\omega, \kappa)$ is $V$-generic.

For a $\kappa$-Universally Baire set $A \subset \omega^{\omega}$, the condition of Theorem 1.4 implies that for $\theta$ large enough and for club many countable elementary submodels $H \prec H(\theta)$,

$$
p[S]^{H[g]}=A \cap H[g]=\mathbb{R}^{H[g]} \backslash p[T]^{H[g]}
$$

whenever $g \subset \operatorname{Col}(\omega, \kappa \cap H)$ is $H$-generic. It is relatively easy to see that an equivalent property is obtained if the trees are replaced by $\operatorname{Col}(\omega, \kappa)$ terms $\dot{A}, \dot{B}$ with the property above. One can either observe that the proof of Theorem 2.1 in [2], asserting that continuous preimages of $A$ in $\kappa^{\omega}$ have the Baire Property, goes through with trivial modification, or one can build the trees directly. First let us fix some notation. We think of a $\operatorname{Col}(\omega, \kappa)$ term for a real as a set $\tau \subset \kappa^{<\omega} \times(\omega \times \omega)$ satisfying $\emptyset \Vdash_{\operatorname{Col}(\omega, \kappa)} \tau \in \omega^{\omega}$, and let $T_{\kappa}$ denote the set of such terms. A name $\dot{A}$ for a set of reals will be a set of pairs $(p, \tau)$ with $p \in \kappa^{<\omega}$ and $\tau \in T_{\kappa}$. For such a name and a submodel $H \prec H(\theta)$ we denote by $\dot{A}\lceil H$ the set $\{(p, \tau \cap H) \mid(p, \tau) \in \dot{A} \cap H\}$. Thus for $g \in(H \cap \kappa)^{\omega}$ we have

$$
\left(\dot{A}\lceil H)_{g}=\pi_{H}(\dot{A})_{\pi_{H}(g)}\right.
$$

where $\pi_{H}$ is the transitivization of $H$. We will typically work with the uncollapsed version for ease of notation.

Definition 1.5. A set of reals $A \subset \mathbb{R}$ is captured at $\kappa$ if there is a name $\dot{A} \in V^{\operatorname{Col}(\omega, \kappa)}$ such that for sufficiently large $\theta$ and for club many $H \in[H(\theta)]^{\omega}$ and every $H$-generic $g \subset \operatorname{Col}(\omega, \kappa \cap H)$,

$$
\left(\dot{A}\lceil H)_{g}=A \cap H[g] .\right.
$$

If $A$ is captured at $\kappa$ and $\dot{B}$ is defined as the set of $(p, \tau)$ such that $\tau$ is a term for a real and $p \Vdash_{\operatorname{Col}(\omega, \kappa)} \tau \notin \dot{A}$, then clearly we have

$$
\emptyset \Vdash_{\mathrm{Col}(\omega, \kappa)} \dot{A}=\mathbb{R} \backslash \dot{B},
$$

so that $\omega^{\omega} \backslash A$ is also captured at $\kappa$. 
Lemma 1.6. $A \subset \omega^{\omega}$ is captured at $\kappa$ if and only if $A \in \Gamma_{\kappa}^{\mathrm{UB}}$.

Proof. Let $f: H(\theta)^{<\omega} \rightarrow H(\theta)$ be such that $C_{f}$ consists of submodels witnessing that $\dot{A}, \dot{B}$ are capturing terms for $A, B$ respectively, where $B$ is the complement of $A$. Let $\psi: \omega \rightarrow \omega^{<\omega}$ be bijective with $\operatorname{ran}(\psi(i)) \subset i$ for all $i$. Define $S \subset \omega^{<\omega} \times H(\theta)^{<\omega} \times\left(\kappa^{<\omega}\right)^{<\omega}$ by $(t, h, p) \in S$ if and only if

(1) $\operatorname{dom}(t)=\operatorname{dom}(h)=\operatorname{dom}(p)$.

(2) $h(0)=(p(0), \tau) \in \dot{A}$.

(3) Each $p(i) \in \operatorname{Col}(\omega, \kappa)$ with $p(i) \subset p(i+1)$, and $p_{i} \Vdash \tau\lceil i=t\lceil i$.

(4) $h(2 i+1)=f(h \circ(\psi(i)))$ and $h(2 i)=p(i)$.

(5) If $h(i) \subset \operatorname{Col}(\omega, \kappa)$ is dense then $p(i) \in h(i)$.

Define $T$ analogously by changing clause $(2)$ to read $h(0)=(p(0), \tau) \in \dot{B}$. If $x \in p[S]$ as witnessed by $(h, p) \in H(\theta)^{\omega} \times \kappa^{\omega}$ then $H=h[\omega]$ is an elementary submodel of $H(\theta)$ which is in the club associated to $\dot{A}$, contains some $(p(0), \tau) \in \dot{A}, g=\{p(i) \mid i<\omega\}$ is $H$-generic for $\operatorname{Col}(\omega, \kappa)$ below $p(0)$, and $t=(\tau \cap H)_{g}$. Thus $t \in A$. Similarly $p[T] \subseteq B$. To see that $S$ and $T$ are absolutely complementing, suppose to the contrary that there are $q$ and $\tau$ with

$$
q \Vdash \tau \notin(p[S] \cup p[T]) .
$$

Then we can find a condition $q^{*}$ below $q$ and a term $\tau^{*}$ such that $q^{*} \Vdash \tau=\tau^{*}$ and $\left(q^{*}, \tau^{*}\right) \in \dot{A} \cup \dot{B}$. Say $\left(q^{*}, \tau^{*}\right) \in \dot{A}$. Let $G \subset \operatorname{Col}(\omega, \kappa)$ be $V$-generic and let $x=\tau_{G}=\tau_{G}^{*}$. In $V[G]$ one easily defines the branch witnessing $x \in p[T]$. This completes the proof.

2. Weak capturing. We now define the pointclass $\Gamma_{\kappa}^{\mathrm{WC}}$ of sets weakly captured at $\kappa$ by requiring only a comeager set of generics for each submodel.

Definition 2.1. Let $\Gamma_{\kappa}^{\mathrm{WC}}$ be the class of sets $A \subset \omega^{\omega}$ for which there exist a term $\dot{A} \in V^{\mathrm{Col}(\omega, \kappa)}$ such that for club many countable elementary $H \prec H(\theta)$ and for comeager many $g \in(H \cap \kappa)^{\omega}$,

$$
\left(\dot{A}\lceil H)_{g}=A \cap H[g] .\right.
$$

If $X$ is a set of ordinals then $\Gamma_{X}^{\mathrm{WC}}$ is defined in the same way.

Note that $\Gamma_{X}^{\mathrm{WC}}=\Gamma_{\kappa}^{\mathrm{WC}}$ whenever $X$ is a set of ordinals of size $\kappa$, and that if $A$ is weakly captured at $\kappa$ then so is $\omega^{\omega} \backslash A$. Clearly $\Gamma_{\kappa}^{\mathrm{UB}} \subseteq \Gamma_{\kappa}^{\mathrm{WC}}$.

We first establish some basic facts about weakly captured sets.

LEMmA 2.2. $\Gamma_{\kappa}^{\mathrm{WC}} \subseteq \Gamma_{\lambda}^{\mathrm{WC}}$ for any infinite cardinals $\kappa$ and $\lambda$ with $\lambda<\kappa$.

Proof. Suppose $\dot{A}$ witnesses $A \in \Gamma_{\kappa}^{\mathrm{WC}}$. Let $\pi: \kappa^{<\omega} \rightarrow \lambda^{<\omega} \times \kappa^{<\omega}$ be a dense embedding. We denote by $\pi(\dot{A})$ the collapse $\operatorname{Col}(\omega, \lambda) \times \operatorname{Col}(\omega, \kappa)$ term corresponding to $\dot{A}$ under the forcing isomorphism generated by $\pi$. We 
now define $\dot{a}$ by $(p, \tau) \in \dot{a}$ if and only if $p \in \operatorname{Col}(\omega, \lambda), \tau \in T_{\lambda}$ and

$$
(p, \emptyset) \Vdash i(\tau) \in \pi(\dot{A})
$$

where $i(\tau)=\{(q, \emptyset, t) \mid(q, t) \in \tau\}$. We claim that $\dot{a}$ witnesses $A \in \Gamma_{\lambda}^{\mathrm{WC}}$. Let $H \prec H(\theta)$ be a submodel which is good for $\dot{A}$ and which contains all relevant objects. Let $C$ be the comeager set of $\operatorname{good} \operatorname{Col}(\omega, \kappa \cap H)$ generics. Thus $\pi[C]$ is comeager in $(\lambda \cap H)^{\omega} \times(\kappa \cap H)^{\omega}$ and for all $(g, h) \in \pi[C]$,

$$
A \cap H[g][h]=\left(\pi(\dot{A})\lceil H)_{(g, h)} .\right.
$$

Thus a Kuratowski-Ulam argument shows that there are comeager many $g \in(\lambda \cap H)^{\omega}$ for which there are comeager many $h \in(\kappa \cap H)^{\omega}$ such that $(g, h) \in \pi[C]$. For any such $g$ we have

$$
\left(\dot{a}\lceil H)_{g}=A \cap H[g]\right.
$$

as desired.

LEMma 2.3. $\Gamma_{\omega}^{\mathrm{WC}}=\Gamma_{\omega}^{\mathrm{UB}}$

Proof. Suppose $f: \omega^{\omega} \rightarrow \omega^{\omega}$ is continuous. Then there is a term $\tau \in T_{\omega}$ such that $f(g)=\tau_{g}$ for every $g \in \omega^{\omega}$. It is easy to see that $M$ defined as the set of $g \in \omega^{\omega}$ such that for some $n$,

$$
g\left\lceil n \Vdash_{\operatorname{Col}(\omega, \omega)} \tau \in \dot{A},\right.
$$

is the open set witnessing that $f^{-1}(A)$ has the Baire Property. To see this let $H$ be a good submodel and $C$ the set of good $H$-generics. Then $f^{-1}(A) \triangle$ $M \subset \omega^{\omega} \backslash C$ is meager.

We now turn to the main theorem. Given $\tau \in T_{\kappa}$ and a set $\sigma$ we denote by $\tau\left\lceil\sigma\right.$ the term $\tau \cap\left(\sigma^{<\omega} \times(\omega \times \omega)\right)$. Note that $\tau\lceil\sigma$ is typically a $\operatorname{Col}(\omega, \sigma)$ term for a real in the sense that this is true for a club of $\sigma \in[\kappa]^{\omega}$. Henceforth we use the quantifier $\forall^{*}$ to mean "for a comeager set".

Definition 2.4. For a set of reals $A$, a condition $p \in \operatorname{Col}(\omega, \kappa)$, and a term for a real $\tau \in T_{\kappa}$ we denote by $S_{p, \tau}^{A}$ the set of $\sigma \in[\kappa]^{\omega}$ such that $\forall^{*} g \in \sigma^{\omega} \quad p \subset g \Rightarrow\left(\tau\lceil\sigma)_{g} \in A\right.$.

Note that $p \subseteq q$ implies $S_{p, \tau}^{A}$ is almost (mod club) contained in $S_{q, \tau}^{A}$. Before presenting our main theorem we mention one subtle point which will arise in the proof (see Theorem 3.2 of [2]).

LEMmA 2.5. Given $\tau \in T_{\kappa}$ the interpretation map $f_{\tau}: \kappa^{\omega} \rightarrow \omega^{\omega}$ defined by $f_{\tau}(g)=\tau_{g}$ is defined and continuous on a comeager set. If $A \in \Gamma_{\kappa}^{\mathrm{UB}}$ then $f_{\tau}^{-1}(A)$ will have the Baire Property in $\kappa^{\omega}$.

Proof. The domain $D$ of $f_{\tau}$ is a comeager $G_{\delta}$ in $\lambda^{\omega}$ and hence there is a homeomorphism $h: \kappa^{\omega} \rightarrow D$. Thus $\left(f_{\tau} \circ h\right)^{-1}(A)$ has the Baire Property in $\kappa^{\omega}$. It follows that $f_{\tau}^{-1}(A)$ has the Baire Property in $D$ and hence in $\kappa^{\omega}$.

ThEOREM 2.6. $\Gamma_{\omega_{1}}^{\mathrm{WC}}=\Gamma_{\kappa}^{\mathrm{WC}}$ under $\mathrm{WRP}_{(2)}(\kappa)$ for any $\kappa \geq \omega_{1}$. 
Proof. Fix $A \in \Gamma_{\omega_{1}}^{\mathrm{WC}}$ and let $B=\omega^{\omega} \backslash A$. Define the terms $\dot{A}, \dot{B}$ by

$$
\begin{aligned}
& \dot{A}=\left\{(p, \tau) \mid p \in \operatorname{Col}(\omega, \kappa), \tau \in T_{\kappa}, S_{p, \tau}^{A} \text { contains a club }\right\}, \\
& \dot{B}=\left\{(p, \tau) \mid p \in \operatorname{Col}(\omega, \kappa), \tau \in T_{\kappa}, S_{p, \tau}^{B} \text { contains a club }\right\}
\end{aligned}
$$

We establish the following:

(1) $(p, \tau) \notin \dot{A} \Rightarrow \exists q \leq p \quad S_{q, \tau}^{B}$ is stationary.

(2) $S_{p, \tau}^{A}$ either contains or is disjoint from a club.

(3) $(p, \tau) \notin \dot{A} \Rightarrow \exists q \leq p \quad(q, \tau) \in \dot{B}$.

(4) $p \Vdash_{\mathrm{Col}(\omega, \kappa)} \tau \in \dot{A}$ implies $(p, \tau) \in \dot{A}$.

Toward (1) assume $(p, \tau) \notin \dot{A}$. Thus there is a stationary set $S \subset[\kappa]^{\omega}$ such that $\sigma \in S$ implies

$$
\neg\left(\forall^{*} g \quad p \subset g \Rightarrow\left(\tau\lceil\sigma)_{g} \in A\right) .\right.
$$

By Lemmas 2.2 and 2.3 there is for each $\sigma \in S$ a condition $q^{\sigma}$ extending $p$ with $q^{\sigma} \subset \sigma$ such that

$$
\forall^{*} g \quad q^{\sigma} \subset g \Rightarrow\left(\tau\lceil\sigma)_{g} \in B .\right.
$$

The point is that $A \in \Gamma_{\sigma}^{\mathrm{UB}}$ as $\sigma$ is countable, and the map $g \rightarrow\left(\tau\lceil\sigma)_{g}\right.$ is continuous on a comeager set. By pressing down we may assume that there is a condition $q$ such that $q^{\sigma}=q$ for any $\sigma \in S$. Thus $S_{q, \tau}^{B}$ is stationary.

To prove (2) assume to the contrary that $S_{p, \tau}^{A}$ and its complement are stationary. From (1) we get a $q$ extending $p$ such that $S_{q, \tau}^{A}$ and $S_{q, \tau}^{B}$ are both stationary. Thus their stationarity reflects to some $X \in[\kappa]^{\omega_{1}}$ with $q \in X$. It is easy to see that

$$
\emptyset \Vdash_{\operatorname{Col}(\omega, X)} \tau\left\lceil X \in \omega^{\omega} .\right.
$$

By assumption there are $\operatorname{Col}(\omega, X)$ terms $\dot{a}, \dot{b}$ which witness that $A \in \Gamma_{X}^{\mathrm{WC}}$. Suppose without loss of generality that there exists $r \in X^{<\omega}$ such that $q \subseteq r$ and

$$
r \Vdash_{\operatorname{Col}(\omega, X)}(\tau\lceil X) \in \dot{a} .
$$

Thus there is a countable $H \prec H(\theta)$ containing $X, r, \tau, \dot{a}, \dot{b}$ such that

$$
\begin{array}{ll}
\forall^{*} g \in[X \cap H]^{\omega} & (\dot{a} \cap H)_{g}=a \cap H[g], \\
\forall^{*} g \in[X \cap H]^{\omega} & q \subset g \Rightarrow(\tau \uparrow(H \cap X))_{g} \in B, \\
\forall^{*} g \in[X \cap H]^{\omega} & r \subset q \Rightarrow H[g] \models\left(\left(\tau\lceil X)_{g} \in \dot{a}_{g}\right) .\right.
\end{array}
$$

Any $H$ in the club associated to $\dot{a}$ such that $H \cap X \in S_{p, \tau}^{B}$ will work. Taking $g \in[H \cap X]^{\omega}$ which is $H$-generic for $\operatorname{Col}(\omega, X)$ below $r$ and which belongs to each of the three comeager sets above gives the desired contradiction.

Toward (3) assume that $(p, \tau) \notin \dot{A}$. By (1) there is $q$ extending $p$ such that $S_{q, \tau}^{B}$ is stationary. By $(2), S_{q, \tau}^{B}$ must contain a club, so that $(q, \tau) \in \dot{B}$ by definition. 
To prove (4) assume that $p \Vdash_{\operatorname{Col}(\omega, \kappa)} \tau \in \dot{A}$ but $(p, \tau) \notin \dot{A}$. Thus there is $q \leq p$ such that $(q, \tau) \in \dot{B}$. Since $q$ also forces $\tau \in \dot{A}$, there is $r$ extending $q$ and $\bar{\tau}$ such that $(r, \bar{\tau}) \in \dot{A}$ and $r \Vdash_{\operatorname{Col}(\omega, \kappa)} \tau=\bar{\tau}$. Passing to a submodel $H$ with $H \cap \kappa \in S_{r, \tau}^{B} \cap S_{r, \bar{\tau}}^{A}$ and an $H$ generic below $r$ in the relevant comeager sets gives a contradiction. We may now verify that $\dot{A}$ witnesses $A \in \Gamma_{\kappa}^{\mathrm{WC}}$. Otherwise by a pressing down argument there is, for $\theta$ large enough, a stationary set $S$ of countable $H \prec H(\theta)$ and a pair $(p, \tau)$ such that $H \in S$ implies $p, \tau \in H$ and

$$
\forall^{*} g \quad p \subset g \Rightarrow \neg\left(\left(\tau\lceil H)_{g} \in(\dot{A} \cap H)_{g} \Leftrightarrow\left(\tau\lceil H)_{g} \in A\right) .\right.\right.
$$

We consider two cases. If $p \Vdash_{\operatorname{Col}(\omega, \kappa)} \tau \in \dot{A}$ then by $(*)$ we have

$$
\forall^{*} g \quad p \subset q \Rightarrow\left(\tau\lceil H)_{g} \notin A,\right.
$$

yet by (3) we have $(p, \tau) \in \dot{A}$, which gives a contradiction. Otherwise there is $q$ extending $p$ such that $q \Vdash_{\mathrm{Col}(\omega, \kappa)} \tau \notin \dot{A}$. By (2) we then have $r$ extending $q$ such that $(r, \tau) \in \dot{B}$ and by $(*)$ we have

$$
\forall^{*} g r \subset q \Rightarrow(\tau \mid H)_{g} \in A,
$$

giving a similar contradiction.

In case the set in question is a function $f \subset \omega^{\omega} \times \omega^{\omega}$, we will have (by an extra argument), for club many $H \prec H(\theta)$ and comeager many $g \subset \operatorname{Col}(\omega, \kappa \cap H)$ which are $H$-generic,

$$
f\lceil H[g] \in H[g] .
$$

We now turn our attention to the Universal Baire Property.

Definition 2.7. A semiscale on a set $A \subset \omega^{\omega}$ is a sequence $\left(A_{n}\right)$ with each $A_{n} \subset \omega^{\omega} \times \omega^{\omega}$ a prewellordering of $A$ such that the associated norms $\left(\phi_{n}\right)$ satisfy the following condition. Suppose $\left(x_{m}\right)$ is a sequence in $A$ and $x \in \omega^{\omega}$ such that $\left(x_{m}\right)$ converges to $x$ and for each $n$ the sequence of ordinals $\left(\phi_{n}\left(x_{m}\right) \mid m<\omega\right)$ is eventually constant. Then $x \in A$.

If $\vec{\phi}=\left(\phi_{n}\right)$ are the norms of such a semiscale then $A$ is the projection of the associated tree $T_{\vec{\phi}}=\left\{\left(x \mid n,\left(\phi_{i}(x)\right)_{i<n}\right) \mid n<\omega, x \in A\right\}$.

Lemma 2.8. If $A \in \Gamma_{\kappa}^{\mathrm{WC}}$ and both $A$ and $\omega^{\omega} \backslash A$ have semiscales whose prewellorderings belong to $\Gamma_{\kappa}^{\mathrm{WC}}$, then $A \in \Gamma_{\kappa}^{\mathrm{UB}}$.

Proof. Let $B$ denote the complement of $A$, let $\dot{A}$ witness that $A \in \Gamma_{\kappa}^{\mathrm{WC}}$ and let $\left(A_{n}\right)$ be the semiscale on $A$ with associated capturing terms $\left(\dot{A}_{n}\right)$. It is straightforward to show that

$$
\emptyset \Vdash_{\mathrm{Col}(\omega, \kappa)}\left(\dot{A}_{n}\right) \text { is a semiscale on } \dot{A} \text {. }
$$


Thus there is a term $\dot{T}$ which is forced to be the tree of $\left(\dot{A}_{n}\right)$. We claim that there is a tree $T_{A} \in V$ such that

$$
\emptyset \Vdash_{\operatorname{Col}(\omega, \kappa)} T_{A}=\dot{T} .
$$

Let $H \prec H(\theta)$ be a countable elementary submodel which contains everything relevant and belongs to the relevant clubs. Suppose toward a contradiction that there are conditions $p, q \in H$ such that

$$
p \Vdash(a, b) \in \dot{T} \quad \text { and } \quad q \Vdash(a, b) \notin \dot{T}
$$

where $a \in \omega^{<\omega}, t \in \theta^{<\omega}$. We may assume $p, q$ have common domain $n<\omega$. Let $g, h$ be $H$-generics which belong to every relevant comeager set, and such that $H[g]=H[h]$. This is accomplished by choosing $g, h$ inductively so that $p \subset g, q \subset h$ and $g, h$ agree on $\omega \backslash n$. We will have $(\dot{T} \cap H)_{g}=$ $(\dot{T} \cap H)_{h}$, which is a contradiction. Thus there is a tree $T_{A} \in H$ such that $H \models\left(\Vdash_{\mathrm{Col}(\omega, \kappa)} T_{A}=\dot{T}\right)$ and by elementarity $T_{A}$ will have the same property in $V$. Repeating the argument for $B$ gives a tree $T_{B}$ such that $T_{A}, T_{B}$ are absolutely complementing, as desired.

Thus under $\mathrm{WRP}_{2}(\kappa)$, self-justifying systems which are weakly captured at $\omega_{1}$ are $\kappa$-Universally Baire. By arguments of [2] this means that they have various regularity properties in the case $\kappa \geq \omega_{2}$.

Corollary 2.9. Assume $\mathrm{WRP}_{(2)}(\kappa)$ and that $A \in \Gamma_{\omega_{1}}^{\mathrm{WC}}$ and both $A$ and $\omega^{\omega} \backslash A$ have semiscales whose prewellorderings belong to $\Gamma_{\omega_{1}}^{\mathrm{WC}}$. Then $A \in \Gamma_{\kappa}^{\mathrm{UB}}$. If $\kappa \geq \omega_{2}$ then $A$ is Lebesgue measurable and has the Ramsey and Bernstein Properties.

Proof. Todorcevic has shown that $\operatorname{WRP}_{(2)}\left(\omega_{2}\right)$ implies $2^{\omega} \leq \omega_{2}$ (see [8]), so that under this principle, sets of reals satisfying the hypotheses of the corollary are all $2^{\omega}$-Universally Baire (and $2^{\omega}$ is regular) by Theorem 2.6, and hence have the regularity properties as in [2].

It also follows that under MM plus two Woodin cardinals, if a set $A$ of reals belongs to an $\omega_{1}$-Universally Baire self-justifying system then $A$ is determined (see Thm. 5.4 of [2]). This raises the following question.

Question 2.10. Assume MM or the generic supercompactness of $\omega_{2}$. Are Universally Baire self-justifying systems determined?

As mentioned in the introduction, it will be shown in [9] that $\operatorname{WRP}_{(2)}\left(\omega_{2}\right)$ cannot imply $\Gamma_{\omega_{1}}^{\mathrm{UB}}=\Gamma_{\omega_{2}}^{\mathrm{UB}}$. We mention this result now.

Theorem 2.11 (Todorcevic). Assume MM. Then any $A \subset \omega^{\omega}$ of size $\omega_{1}$ is $\omega_{1}$-Universally Baire but not $\omega_{2}$-Universally Baire. 
This together with Theorem 2.6 shows that weak capturing is indeed weaker than capturing under MM.

\section{Corollary 2.12. Assume MM. Then $\Gamma_{\omega_{2}}^{\mathrm{WC}} \neq \Gamma_{\omega_{2}}^{\mathrm{UB}}$.}

The following is about all reflection gives and may be useful in another context.

Lemma 2.13. Assume $\operatorname{WRP}_{(2)}\left(\omega_{2}\right)$ and that $A \subset \omega_{2}^{\omega}$ is such that $A \cap \delta^{\omega}$ has the Baire Property in $\delta^{\omega}$ for every $\delta<\omega_{2}$. Then for every $p \in \omega_{2}^{<\omega}$ there is $q \in \omega_{2}^{<\omega}$ with $p \subseteq q$ such that $A \cap \sigma^{\omega}$ is comeager below $q$ in $\sigma^{\omega}$ for a club of $\sigma \in\left[\omega_{2}\right]^{\omega}$ or $A \cap \sigma^{\omega}$ is meager below $q$ in $\sigma^{\omega}$ for a club of $\sigma \in\left[\omega_{2}\right]^{\omega}$

Proof. Note that we do not necessarily assume that $A$ is the continuous preimage of a set of reals. If $A \cap \sigma^{\omega}$ is meager below $p$ for a club of $\sigma$ we are done. Thus we may assume by pressing down that there are disjoint stationary sets $S_{1}$ and $S_{2}$ and a fixed $q$ extending $p$ such that $A \cap \sigma^{\omega}$ is meager below $q$ if $\sigma \in S_{1}$ and comeager below $q$ if $\sigma \in S_{2}$. These sets reflect to some $[\delta]^{\omega}$. Suppose without loss of generality that player I wins the Banach-Mazur game $G^{* *}\left(A \cap \delta^{\omega}\right)$, which is determined by hypothesis. It follows that a club of $\sigma \in[\delta]^{\omega}$ are closed under this winning strategy. By selecting such a $\sigma$ in $S_{2}$ we reach a contradiction.

If more of generic compactness is used, the hypothesis of weakly captured scales can be avoided. Recall from [5] the Game Reflection Principle $\mathrm{GRP}^{+}$. For a set $\mathcal{A} \subset \theta^{<\omega_{1}}$ the game $G(\mathcal{A})$ has two players who alternate playing ordinals below $\theta$ for $\omega_{1}$ rounds. Player II wins if the cooperative play stays in $\mathcal{A}$ at every countable stage. For a set $H \subset \theta$, the game $G^{H}(\mathcal{A})$ is the same except both players are restricted to playing ordinals from $H$. Now, $\mathrm{GRP}^{+}$asserts that II wins $G(\mathcal{A})$ if and only if II wins $G^{H}(\mathcal{A})$ for an $\omega_{1}$-club of $H \in[\theta]^{\omega_{1}}$.

TheOREM 2.14 (with S. Todorcevic). $\mathrm{GRP}^{+}$implies $\Gamma_{\omega_{1}}^{\mathrm{UB}}=\Gamma_{\infty}^{\mathrm{UB}}$.

Proof. Suppose $A \subset \theta^{\omega}$ is a continuous preimage of a set in $\Gamma_{\omega_{1}}^{\mathrm{UB}}$. Thus $A \cap H^{\omega}$ has the Baire Property in $H^{\omega}$ for any $H \in[\theta]^{\omega_{1}}$. We describe a game which is easily coded as one of the forms described above. I and II alternate playing elements of $\theta^{<\omega}$ for $\omega$ rounds producing a strictly increasing sequence $\left(p_{i} \mid i \in \omega\right)$. At II's first move however he must declare whether the resulting play $p=\bigcup p_{i} \in \theta^{\omega}$ will belong to $A$ or not. If he declares that $p \in A$ then he plays $p_{1}=p_{0}$ at this turn only. II wins if his prediction is correct.

3. Further remarks. It is of course consistent that there be a selfjustifying system which is $\omega_{1}$-Universally Baire but not Universally Baire. For a heavy-handed example, suppose there exist infinitely many Woodin 
cardinals. Then the pointclass of projective sets $\Gamma$ is itself a self-justifying system and $\Gamma \subset \Gamma_{\omega_{1}}^{\mathrm{UB}}$. But if $V=L[X]$ for some set $X$, which may be assumed, then by Thm. 3.4 of [2] there is a $\Sigma_{2}^{1}$ set which cannot be Universally Baire (otherwise $X^{\#}$ exists). An example of perhaps the lowest complexity would be $\Delta_{2}^{1}$, which can be arranged from less than a Mahlo cardinal. One can still ask whether it is consistent for there to be a definable set of reals which is weakly captured but not Universally Baire, or to what extent weak capturing is a regularity property.

QuESTION 3.1. Is it consistent for there to be a projective set $A \in$ $\Gamma_{\kappa}^{\mathrm{WC}} \backslash \Gamma_{\kappa}^{\mathrm{UB}}$ with $\kappa \in\left\{\omega_{1}, \omega_{2}\right\}$ ?

Question 3.2. Suppose $\kappa \geq 2^{\omega}$ is regular and $A \in \Gamma_{\kappa}^{\mathrm{WC}}$. Must $A$ have other regularity properties?

The answer to both questions is very likely no. The second question can likely be answered in the negative under MM. Regarding the first we conjecture that it is consistent for there to be a $\Sigma_{2}^{1}$ counterexample. First note that $\Delta_{2}^{1}$ cannot be a counterexample.

Lemma 3.3. $\Delta_{2}^{1} \subseteq \Gamma_{\omega_{1}}^{\mathrm{WC}}$ implies $\Delta_{2}^{1} \subseteq \Gamma_{\omega_{1}}^{\mathrm{UB}}$.

The point is that if $A$ is $\Delta_{2}^{1}$ then the norms of the $\Sigma_{2}^{1}$ scale on $A$ are also $\Delta_{2}^{1}$. Thus Lemma 2.8 applies. Moreover $\Delta_{2}^{1} \subset \Gamma_{\omega_{1}}^{\mathrm{WC}}$ under $\operatorname{WRP}_{(2)}\left(\omega_{2}\right)$ implies $\Delta_{2}^{1} \subset \Gamma_{\omega_{2}}^{\mathrm{UB}}$. Note that by the proof Theorem 3.3 of [2], and Lemma 3.3 above, $\Delta_{2}^{1}$ weak capturing at $\omega_{1}$ implies $\omega_{1}$ is inaccessible in $L$. However we cannot show that even the statement "all projective sets are weakly captured at every $\kappa$ " has greater strength. The point is that at $\Sigma_{2}^{1}$ anyway (it is open if this persists) there is an equivalence between the Universal Baire Property and generic absoluteness which does not seem to follow from weak capturing. This leads us to believe that the divergence between the two notions occurs at this level. It has been known since [10] that generic absoluteness can be used to violate covering. The following lemma is implicit in [2].

LEMMA 3.4. $\Sigma_{2}^{1} \subseteq \Gamma_{\kappa}^{\mathrm{UB}}$ if and only if for sufficiently large $\theta$ there is a club of countable $X \prec H(\theta)$ such that $X[g] \prec_{\Sigma_{2}^{1}} V$ for any $X$-generic $g \subset \operatorname{Col}(\omega, X \cap \kappa)$.

Theorem 3.5. Suppose $\Sigma_{2}^{1} \subseteq \Gamma_{\omega_{1}}^{\mathrm{UB}}$. Let $x \in \omega^{\omega}$. Then there is a club of $\alpha<\omega_{1}$ which are regular cardinals of $L[x]$. Further, $\omega_{1}^{V}$ is weakly compact in $L[x]$.

Proof. For simplicity assume $x \in L$. Suppose to the contrary that there is a stationary set $S \subset \omega_{1}$ such that every $\alpha$ in $S$ is not a regular cardinal of $L$. Let $X$ be a countable elementary submodel of $H(\theta)$ such that $X \cap \omega_{1}$ 
$=\alpha \in S$. Let $H$ be the transitivization of $X$. So $H \models\left(\alpha=\omega_{1}\right)$ and hence $H$ thinks that $\alpha$ is a regular cardinal of $L$. In $V$ let $g \subset \operatorname{Col}(\omega, \alpha)$ be $H$-generic, and let $y \in H[g]$ be a real coding a well-ordering of length $\alpha$. The statement "some $L_{\gamma}[y]$ thinks that the ordinal coded by $y$ is not a regular cardinal of $L^{\prime \prime}$ is $\Sigma_{2}^{1}(y)$ and hence true in $H[g]$. Thus some level of $L^{H[g]}=L^{H}$ thinks that $\alpha$ is not a regular cardinal, a contradiction. It follows that $\omega_{1}^{V}$ is Mahlo in $L$. Finally, we show that $\omega_{1}^{V}$ has the tree property in $L$. Suppose $T \in L$ is an $\omega_{1}^{V}$ tree. Then for stationary many $\alpha<\omega_{1}^{V}, T \cap L_{\alpha}$ is an $\alpha$-tree in $L$. Let $X \prec H(\theta)$ be as before with $X \cap \omega_{1}$ such an $\alpha$. Some $L_{\beta}$ sees a branch through $T \cap L_{\alpha}$ and using absoluteness again we conclude that $X$ contains such a branch.

Pushing the argument above, one can show that $\omega_{1}^{V}$ is Mahlo to ineffables in $L$ and more. On the other hand, the closure of $H\left(\omega_{2}\right)$ under sharps ensures $\Sigma_{2}^{1} \subseteq \Gamma_{\omega_{1}}^{\mathrm{UB}}$. But in the presence of $\mathrm{WRP}_{2}\left(\omega_{2}\right)$, by Woodin's result mentioned in the Introduction, $H\left(\omega_{3}\right)$ would be closed under sharps, which in turn implies $\Sigma_{2}^{1} \subseteq \Gamma_{\omega_{2}}^{\mathrm{UB}}$. This is an obstacle to using Theorem 2.6 to arrange a definable set which is weakly captured at $\omega_{2}$ but not $\omega_{2}$-Universally Baire. Should such an example at the level of $\Sigma_{2}^{1}$ be possible it may require answering 3.7 below.

Question 3.6. Does all projective sets weakly captured imply $\omega_{1}$ is weakly compact in $L$ ?

QUESTION 3.7. What is the consistency strength of $\Sigma_{2}^{1} \subseteq \Gamma_{\omega_{1}}^{\mathrm{UB}}$ ?

\section{References}

[1] M. Bekkali, Topics in Set Theory, Lecture Notes in Math. 1476, Springer, 1991.

[2] Q. Feng, M. Magidor and H. Woodin, Universally Baire sets of reals, in: Set Theory of the Continuum (Berkeley, CA, 1989), Math. Sci. Res. Inst. Publ. 26, Springer, 1992, 203-242.

[3] M. Foreman, M. Magidor and S. Shelah, Martin's maximum, saturated ideals and nonregular ultrafilters, I, Ann. of Math. (2) 127 (1988), 1-47.

[4] A. Kechris, Classical Descriptive Set Theory, Grad. Texts in Math. 156, Springer, 1995.

[5] B. König, Generic compactness reformulated, Arch. Math. Logic 43 (2004), 311-326.

[6] T. Jech, Multiple Forcing, Cambridge Univ. Press, 1986

[7] J. Steel and S. Zoble, $\operatorname{SRP}\left(\omega_{2}\right)$ implies $\mathrm{AD}^{L(\mathbb{R})}$, in preparation.

[8] S. Todorcevic, Reflection of stationary sets I, handwritten notes, Sept. 1984.

[9] S. Todorcevic and S. Zoble, Baire reflection, in preparation.

[10] W. H. Woodin, On the consistency strength of projective uniformization, in: Logic Colloquium '81 (Proc. Herbrand Sympos., Marseille, 1981), J. Stern (ed.), Stud. Logic Found. Math. 107, North-Holand, 1982, 365-384. 
[11] W. H. Woodin, The Axiom of Determinacy, Forcing Axioms, and the Nonstationary Ideal, de Gruyter Ser. Logic Appl. 1, de Gruyter, 1999.

Department of Mathematics

University of Toronto

Toronto, Ontario

M5S 2E4, Canada

E-mail: szoble@math.toronto.edu

Received 26 August 2005 